\title{
Cataract surgery in the face of ocular surface disease
}

Authors: Asadolah Movahedan, Ali R. Djalilian

Affiliations: Department of Ophthalmology and Visual Sciences, University of Illinois at Chicago, 1855 W Taylor Street, Chicago, IL 60612

Corresponding author: Ali R. Djalilian, MD.

Department of Ophthalmology and Visual Sciences, University of Illinois at

Chicago, 1855 W. Taylor St, M/C 648, Chicago, IL 60612.

Phone: 312-996-8936

E-mail address: adjalili@uic.edu 


\section{Abstract}

\section{Purpose of review}

This paper reviews the importance of ocular surface management in patients undergoing cataract surgery. The current strategies for the diagnosis and management of ocular surface disease in cataract surgery patients are discussed.

\section{Recent findings}

The current trend is to diagnose and treat ocular surface disease before cataract surgery using a stepwise regimen tailored to the individual patient and disease severity.

\section{Summary}

Maintaining a healthy ocular surface is essential for achieving the best visual outcome in cataract patients. Ocular surface preparation is beneficial not only in patients with established ocular surface disease, but also in those with minimal signs or symptoms of surface disease.

\section{Key words}

Ocular surface disease, cataract surgery, dry eye, blepharitis, lid margin disease 


\begin{abstract}
Abbreviations
ST: Schirmer test, TBUT: Tear film break up time, HPMC: Hydroxypropyl methylcellulose, NSAIDs: Non-steroidal anti inflammatory drugs
\end{abstract}

\title{
Introduction
}

Ocular surface disease, particularly dry eye syndrome, is one of the most common conditions in the elderly $[1,2]$. Since age-related cataract constitutes most of patients undergoing phacoemulsification, the diagnosis and management of ocular surface disease is therefore indispensible in majority of these patients. In a recent multi-center study, presented at American Society of Cataract and Refractive Surgery, more than half of all patients undergoing cataract surgery were found to have significant dry eye disease based on objective testing (Trattler WB,et al., unpublished data). This is important since the incidence and severity of dry eye symptoms increase even further after cataract surgery $[3,4]$. In particular, phacoemulsification has been shown to reduce tear meniscus height and tear break up time $[5,6]$ and increase squamous metaplasia on conjunctival impression cytology [7]. The mechanism for the exacerbation of surface disease after cataract surgery likely includes several factors: increased inflammatory mediators due to post-operative inflammation, toxicity from the use of benzalkonium chloride containing eye drops, and damage to the corneal nerves from limbal incisions. The presence and exacerbation of ocular surface disease may have special importance in patients receiving presbyopic intraocular lenses since disturbances in the tear film can significantly impact the visual quality and patient satisfaction postoperatively. In addition to having delayed visual recovery after cataract surgery, patients with moderate to severe ocular surface disease are also at 
higher risk of post-operative complications, namely infections and corneal melts. Therefore, aggressive management of the ocular surface disease is imperative in all cataract surgery patients. This review summarizes the latest approaches to the diagnosis and management of ocular surface disease in the setting of cataract surgery.

\section{Diagnosis}

Pre-operative recognition of patients with ocular surface disease provides an opportunity to optimize the surface before proceeding with surgery. The patient history is often one of the most important elements in the diagnosis of patients with dry eyes or "dysfunctional tear syndrome" $[8,9]$. Besides the usual symptoms of grittiness or discomfort, blurry vision that is worsened by visual activity, can sometimes be overlooked as a symptom of dry eye and could be mistakenly attributed to the patient's cataract [10]. As noted by others, fluctuating vision either before or after cataract surgery is almost always a sign of tear film insufficiency [11]. Likewise, history of a systemic collagen vascular diseases or associated manifestations such as arthritis or dry mouth provides important clues for the possibility of concomitant ocular surface disease.

\section{Clinical examination and diagnostic testing}

The clinical exam of the cataract surgery patient can provide additional clues that suggest tear film insufficiency. These include findings such as debris in the tear film, a low tear meniscus height, evidence of lid margin disease, and conjunctival inflammation.

Diagnostic testing is greatly valuable both for the detection of early changes due to dry eyes and also to grade the severity of surface disease. The most commonly performed tests include 
Schirmer test (ST), tear breakup time (TBUT), and ocular surface staining. It is noteworthy that clinical findings and diagnostic tests are only weakly associated with patient symptoms in ocular surface disease $[12,13]$.

There is an ongoing debate about the reproducibility and sensitivity of Schirmer test since it measures reflex tearing and the stimulus is not standardized. Serin et al. [14] has suggested that performing the test without anesthesia with the eyes closed produces less variable results and increases the repeatability of the test. Two-minute ST has been proposed as an acceptable alternative for 5-minute test both for the patient and examiner; Overall, ST may be less useful in mild cases and instead more applicable for detecting patients with moderate to severe aqueous tear deficiency.

A TBUT of less than 10 seconds is likewise highly suggestive of an inadequate tear film and is a valuable diagnostic test when looking for evidence of ocular surface disease pre-operatively.

\section{Ocular surface staining}

Corneal staining with fluorescein is a widely used diagnostic modality to assess the severity of the dry eyes. (Fig.1) Lisamine green and Rose Bengal, stain epithelial cells that have lost their mucin covering; both are more sensitive than fluorescein in detecting early signs of tear film insufficiency by looking for staining in the nasal and temporal conjunctiva.

Hardten [15] has suggested using a test he calls "ocular surface stress test" to identify high-risk patients for developing dry eye signs and symptoms after phacoemulsification. "After the dilating drops and anesthetic, the patient is directed to sit in the waiting room for 30-60 minutes and is then reexamined. If irregular epithelium or punctate keratopathy is observed during this 
follow-up examination, that patient probably has problems maintaining a healthy epithelial surface after surgery.” [page 855]

\section{Corneal Sensation}

Neurotrophic corneal disease is relatively common and especially in diabetic patients is a frequent cause of chronic surface disease [16]. The use of cotton wisp to measure sensation can identify such patients. While neurotrophic patients are less likely to complain of the typical dry eye symptoms (other than fluctuating or decreased vision), they are at particularly higher risk for ocular surface complications such as persistent epithelial defects following cataract surgery.

\section{Management}

Given the elective nature of cataract surgery, once a patient has been identified as having poorly controlled ocular surface disease, surgery is typically postponed until the surface can be optimized.

\section{Pre-operative care}

Management of ocular surface disease may be done most effectively by following established treatment guidelines [17]. In this step-wise approach, treatment begins with artificial tears which have been shown to diminish ocular symptoms and improve vision-related function and dry eye signs (TBUT) in the majority of cataract surgery patients [18].

Anti inflammatory agents play a major role in treatment of moderate to severe dry eye due to critical role of inflammation in pathogenesis of the ocular surface disease. Topical 
corticosteroids and immunoregulatory agents constitute the main therapies currently in use. Several studies have noted the efficacy of topical corticosteroids in treatment of dry eye [19]. Steroids have a rapid onset of action and therefore, are helpful in circumstances that immediate response is needed. In a randomized, double-masked, placebo-controlled study in keratoconjunctivitis sicca patients, a significant difference between the loteprednol etabonate ophthalmic suspension $0.5 \%$-treated group and vehicle-treated group was found in patients with moderate clinical inflammation after 2 weeks of therapy. In addition, steroid-treated patients retained their improvement compared with control group [20].

Cyclosporine has gained popularity because its long term use is devoid of steroid induced side effects. Although, it may take several weeks of treatment in order to get a therapeutic effect. The mechanism of action of Cyclosporine in increasing tear production is not fully understood, but the drug seems to have immunomodulatory effects that reduce inflammation. Pflugfelder $e t$ al. [21] showed a significant increase in goblet cell density in the bulbar conjunctiva in patients with dry eye with $0.05 \%$ Cyclosporine emulsion therapy twice a day for 12 weeks comparing the same treatment with artificial tears. Sahli et al. [22] reported the beneficial effects of topical Cyclosporine therapy twice daily in increasing ST values and TBUT scores and improvement of cytological grade of dry eye comparing the patients before, and 6 months after treatment. Donnenfeld et al. [23**] found that Cyclosporine $0.05 \%$ therapy improves visual quality after multifocal intraocular lens implantation suggesting its beneficial effect on tear-film quality. A Recent study showed that decreasing therapy to once daily for patients controlled with topical $0.05 \%$ Cyclosporine twice daily for at least 1 year, still may allow suppression of the dry eye disease [24*]. An important consideration in patients with cataract surgery is to maximally 
suppress inflammation pre-operatively given that inflammation increases significantly after surgery.

In patients with significant aqueous deficiency, consideration should be given to punctual occlusion. Punctal plugs have been shown to improve vision in dry eye patients by stabilizing tear film through decreasing its osmolarity [25]. Combining punctual plugs with $0.05 \%$ Cyclosporine is shown to result in the best ST scores, rose Bengal staining, and reduction in overall artificial tear use compared to either treatments alone [26]. Punctal occlusion is best done after controlling the ocular surface inflammation.

Management of lid disease is likewise essential for optimization of surgical outcomes. In one series, blepharitis was the number one reason for cancellation of cataract surgery [27] as it is thought to be a primary risk factor for endophthalmitis [28]. Pre-operative treatment begins with a prolonged commitment to eyelid hygiene. Topical antibiotics are used to control Staphylococcal growth on the eyelids. Recent antibiotics such as topical azithromycin are promising in unresponsive patients and have been shown to effectively reduce signs and symptoms of blepharitis [29]. If severe inflammation or complications like phlyctenules or severe conjunctivitis are present, topical antibiotic-steroid combinations can be used for short periods of time [30]. Oral antibiotics, commonly doxycycline or minocycline, are reserved for refractory cases most of whom have significant meibomian gland disease or ocular rosacea [31]. Tetracycline drugs improve the symptoms [32] boost TBUT [33] and also decrease eyelid flora. These are typically started at least one month before cataract surgery. Increasing intake of Omega-3 fatty acids from fish oil and flax seed oil may also have anti-inflammatory effects which are beneficial in the management of lid disease. 


\section{Intra-operative care}

Intra-operatively, the ocular surface is prone to damage from preservative containing anesthetics and more importantly from desiccation. Frequent irrigation with balanced salt solution or more viscous eye lubricants or viscoelastics [34] can minimize the desiccating stress to the surface. In a randomized controlled double masked study, hydroxypropyl methylcellulose (HPMC) 2\% gel provided significantly better optical clarity and less frequent need for irrigation compared with Balanced Salt Solution during cataract surgery. One-hour after surgery there was no difference in the corneal staining between the two groups $\left[35^{*}\right]$.

The surgical incision may potentially impact the ocular surface after cataract surgery.

Historically, large incision from extra-capsular cataract extractions induced significant damage to the corneal nerves [36]. However, small incision cataract wounds [37, 38] as well as limbal relaxing incisions seem to induce localized damage to the corneal nerves with subsequent reduced corneal sensation [39]. However, the effect of decreased corneal sensation on ocular surface and tear film has not been studied.

\section{Post-operative care}

After cataract surgery the signs and/or symptoms of ocular surface disease typically get worse. Prolonged use of post-operative medications may be one of the contributing factors. Therefore, in patients with surface disease it is best to stop or taper medications when no longer needed. One particular type of medication commonly used after cataract surgery that deserves special attention is topical non-steroidal anti-inflammatory drug (NSAID). Topical NSAIDs such as nepafenac, ketorolac and diclofenac have been reported to cause corneal melting mainly in the presence of epithelial breakdown [40-42]. These complications may be more likely in patients 
with significant ocular surface disease and dry eye such as Sjogren's syndrome. There is a suggestion that concomitant use of topical steroids with NSAIDs may reduce this complication, however it does not completely prevent it. Therefore, in patients with significant ocular surface disease it is prudent to minimize or even avoid the use of topical NSAIDs especially as a single agent.

\section{Cataract Surgery in Severe Ocular Surface Disease}

As mentioned in the last section, patients with severe ocular surface disease are at significantly greater risk of complication after cataract surgery. One noteworthy complication is sterile corneal melts which has been reported in patients with immune mediated ocular surface diseases such as Sjogren's syndrome, graft versus host disease, and Stevens-Johnson syndrome [42-47]. Optimization of the ocular surface prior to cataract surgery is imperative in these patients. While topical steroids are cautiously used in patients with an unstable surface, systemic immunosuppression may be indicated to suppress inflammation in the setting of systemic disease [48]. Cataract surgery in patients with ocular cicatricial pemphigoid (OCP) requires the disease to be controlled for a minimum of one year while peri-operative use of systemic steroids is highly recommended.

\section{Conclusion}

Given that cataract surgery is a surface damaging event, it is important to consider ocular surface in patients pre-operatively. Ocular surface preparation is beneficial not only in patients with 
established ocular surface disease, but also in those with minimal signs or symptoms of surface disease. Currently, there is more interest among the cataract surgeons to further improve the outcomes of surgery by aggressively treating the ocular surface before and after operation.

\section{Key points}

- Cataract Surgery has clearly been shown to worsen ocular surface disease at least temporarily.

- The current trends, is to vigorously treat ocular disease pre-operatively in patients undergoing cataract surgery

- Patients with moderate to severe ocular surface disease are at increased risk for complications, most notably corneal melting, in the post-operative period. 
Financial Disclosure: None

Conflicts of interest: None declared

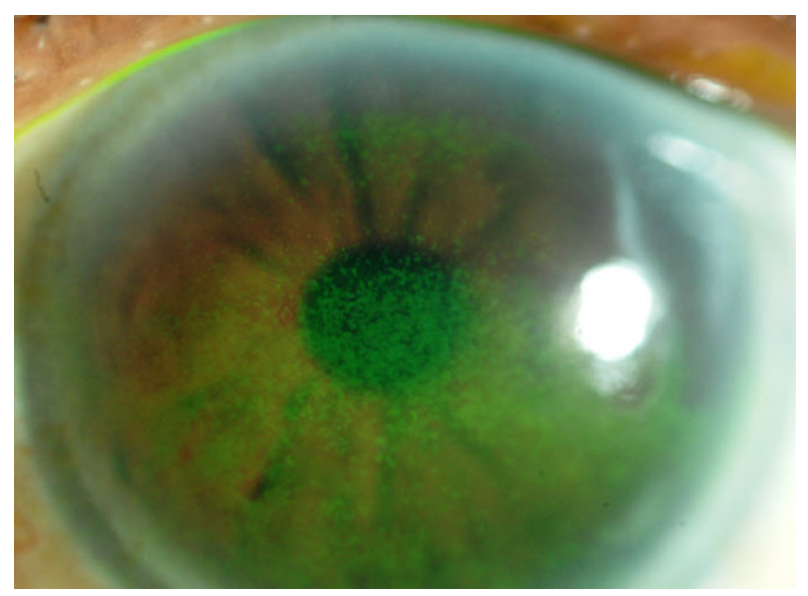

Fig1. Fluorescein corneal staining of a patient with severe ocular surface disease due to graft versus host disease demonstrating significant epitheliopathy after cataract surgery

The patient's surface disease worsened despite pre-operative use of topical Cyclosporine and ultimately required punctual occlusion after surgery. Topical NSAIDs were avoided in this patient to minimize the risk of a corneal melt.

Source: Original figure 


\section{References and recommended reading}

*of special interest

**of outstanding interest

1 Schaumberg DA, Sullivan DA, Buring JE, Dana MR: Prevalence of dry eye syndrome among US women. Am J Ophthalmol 2003, 136:318-26.

2 Han SB, Hyon JY, Woo SJ, et al.: Prevalence of dry eye disease in an elderly Korean population. Arch Ophthalmol 2011, 129:633-8.

3 Cho Yk, Kim MS: Dry eye after cataract surgery and associated intraoperative risk factors. Korean J Ophthalmol 2009, 23: 65-73.

4 Li XM, Hu L, Hu J, Wang W: Clinical observation of dry eye in patients before and after cataract surgery. Zhonghua Yan Ke Za Zhi 2007, 43:10-3.

5 Ram J, Gupta A, Brar G, et al.: Outcomes of phacoemulsification in patients with dry eye. J Cataract Refract Surg 2002, 28:1386-1389.

6 Khanal S, Tomlinson A, Esakowitz L, et al.: Changes in corneal sensitivity and tear physiology after phacoemulsification. Ophthalmic Physiol Opt. 2008, 28:127-34.

7 Li XM, Hu L, Hu J, Wang W: Investigation of dry eye disease and analysis of the pathogenic factors in patients after cataract surgery. Cornea 2007, 26:S16-20.

8 Korb DR: Survey of preferred tests for diagnosis of the tear film and dry eye. Cornea 2000, 19:483-486. 
9 Nichols KK, Nichols JJ, Zadnik K.: Frequency of dry eye diagnostic test procedures used in various modes of ophthalmic practice. Cornea 2000, 19:477-482.

10 Lemp MA: Report of the National Eye Institute/Industry workshop on Clinical Trials in Dry Eyes. CLAO J 1995, 21:221-32.

11Roberts CW, Elie ER: Dry eye symptoms following cataract surgery. Insight 2007, 32:14-21; quiz 22-3.

12 Bjerrum KB: Test and symptoms in keratoconjunctivitis sicca and their correlation. Acta Ophthalmol Scand 1996, 74:436-41.

13 Hay EM, Thomas E, Pal B, et al.: Weak association between subjective symptoms or and objective testing for dry eyes and dry mouth: results from a population based study. Ann Rheum Dis 1998, 57:20-4.

14 Serin D, Karsloğlu S, Kyan A, Alagöz G: A simple approach to the repeatability of the schirmer test without anesthesia: eyes open or closed? Cornea 2007, 26:903-6.

15 Hardten DR: Dry eye disease in patients after cataract surgery. Cornea 2008, 27:855.

16 Lockwood A, Hope-Ross M, Chell P: Neurotrophic keratopathy and diabetes mellitus. Eye (Lond) 2006, 20:837-9.

17 Behrens A, Doyle JJ, Stern L, et al.: Dysfunctional tear syndrome: a Delphi approach to treatment recommendations. Cornea 2006, 25:900-7.

18 Sánchez MA, Arriola-Villalobos P, Torralbo-Jiménez P, et al.: The effect of preservative-free HP-Guar on dry eye after phacoemulsification: a flow cytometric study. Eye 2010, 24:1331-7.

19 Pflugfelder SC: Antiinflammatory therapy for dry eye. Am J Ophthalmol 2004, 137:337-42. 
20 Pflugfelder SC, Maskin SL, Anderson B, et al.: A randomized, double-masked, placebocontrolled, multicenter comparison of loteprednol etabonate ophthalmic suspension, $0.5 \%$, and placebo for treatment of keratoconjunctivitis sicca in patients with delayed tear clearance. Am J Ophthalmol 2004, 138:444-57.

21 Pflugfelder SC, De Paiva CS, Villarreal AL, Stern ME. Effects of sequential artificial tear and cyclosporine emulsion therapy on conjunctival goblet cell density and transforming growth factor-beta2 production. Cornea 2008, 27:64-9.

22 Sahli E, Hoşal BM, Zilelioğlu G, Gülbahçe R, et al.: The effect of topical cyclosporine A on clinical findings and cytological grade of the disease in patients with dry eye. Cornea 2010, 29:1412-6.

**23 Donnenfeld ED, Solomon R, Roberts CW, et al.: Cyclosporine $0.05 \%$ to improve visual outcomes after multifocal intraocular lens implantation. J Cataract Refract Surg 2010, 36:1095100.

** This randomized prospective contralaterally controlled double-masked trial, is one of the most important studies that evaluate the effect of treatment of ocular surface disease in cataract patients. One important feature is that it focuses on visual outcome and patient's satisfaction.

*24 Su MY, Perry HD, Barsam A, Perry AR, et al.: The Effect of Decreasing the Dosage of Cyclosporine A $0.05 \%$ on Dry Eye Disease After 1 Year of Twice-Daily Therapy. Cornea 2011 Mar 14. [Epub ahead of print]

* Decreasing the dosage of topical Cyclosporine with the same therapeutic effect promises improved quality of life and better maintenance for dry eye patients 
25 Gilbard JP, Rossi SR, Azar DT, Heyda KG: Effect of punctal occlusion by Freeman silicone plug insertion on tear osmolarity in dry eye disorders. CLAO J 1989, 15:216-8.

26 Roberts CW, Carniglia PE, Brazzo BG: Comparison of topical cyclosporine, punctal occlusion, and a combination for the treatment of dry eye. Cornea 2007, 26:805-9.

27 Stead RE, Stuart A, Keller J, Subramaniam S: Reducing the rate of cataract surgery cancellation due to blepharitis. Eye (Lond) 2010, 24:742.

28 Speaker MG, Milch FA, Shah MK, et al.: The role of external bacterial flora in the pathogenesis of acute postoperative endophthalmitis. Ophthalmology 1991, 98: 639-649.

29 Luchs J: Efficacy of topical azithromycin ophthalmic solution 1\% in the treatment of posterior blepharitis. Adv Ther 2008, 25:858-870.

30 Jackson WB: Blepharitis, current strategies for diagnosis and management. can $\mathrm{j}$ ophthalmol 2008, 43:170-9.

31 Alvarenga LS, Mannis MJ: Ocular rosacea. Ocul surf 2005, 3:41-58.

32 Akpek EK, Merchant A, Pinar V, Foster CS: Ocular rosacea: patient characteristics and follow-up. Ophthalmology 1997, 104:1863-7.

33 Zengin N, Tol H, Gündüz K, et al.: Meibomian gland dysfunction and tear film abnormalities in rosacea. Cornea 1995, 14:144-6.

34 Lindstrom RL: The effects of blepharitis on ocular surgery. Ocul Surf 2009, 7:S19-20. 
*35 Chen YA, Hirnschall N, Findl O: Comparison of corneal wetting properties

of viscous eye lubricant and balanced salt solution to maintain optical clarity during cataract surgery. J Cataract Refract Surg 2011 Jul 20. [Epub ahead of print]

*Clear view of a surgeon is critical in any ophthalmic surgery. Intra operative BSS irrigation might not always provide the best optical clarity. It also tends to evaporate rapidly thus making the ocular surface prone to desiccation stress. This article suggests using HMPC $2 \%$, addresses both of these disadvantages.

36 Lyne A: Corneal sensitivity after surgery. Trans Ophthalmol Soc UK 1982, 102: 302-305.

37 John T: Corneal sensation after small incision, sutureless, one-handed phacoemulsification. J Cataract Refract Surg 1995, 21:425-8.

38 Kohlhaas M, Stahlhut O, Tholuck J, Richard G: Development of corneal sensitivity after phacoemulsification with scleral tunnel incision. Klin Monbl Augenheilkd 1997, 211:32-6.

39 Moon SW, Yeom DJ, Chung SH: Neurotrophic corneal ulcer development following cataract surgery with a limbal relaxing incision. Korean J Ophthalmol 2011, 25:210-3.

40 Wolf EJ, Kleiman LZ, Schrier A: Nepafenac-associated corneal melt. J Cataract Refract Surg 2007, 33:1974-5.

41 Khalifa YM, Mifflin MD: Keratitis and corneal melt with ketorolac tromethamine after conductive keratoplasty. Cornea 2011, 30:477-8.

42 Gokhale NS, Vemuganti GK: Diclofenac-induced acute corneal melt after collagen crosslinking for keratoconus. Cornea 2010, 29:117-9. 
43 Pfister RR, Murphy GE: Corneal ulceration and perforation associated with Sjögren's syndrome. Arch Ophthalmol 1980, 98:89-94.

44 Cohen KL: Sterile corneal perforation after cataract surgery in Sjögren's syndrome. Br J Ophthalmol 1982, 66:179-82.

45Perez VL, Azar DT, Foster CS: Sterile corneal melting and necrotizing scleritis after cataract surgery in patients with rheumatoid arthritis and collagen vascular disease. Semin Ophthalmol 2002, 17:124-30.

46 van der Meulen IJ, van Rooij J, Hazes JW, et al.: Corneal melting after ocular surgery in patients with auto-immune disorders. Ned Tijdschr Geneeskd 2007, 151:920-3.

47 Sangwan VS, Burman S: Cataract surgery in Stevens-Johnson syndrome. J Cataract Refract Surg 2005, 31:860-2.

48 Cordero-Coma M, Anzaar F, Sobrin L, Foster CS: Systemic immunomodulatory therapy in severe dry eye secondary to inflammation. Ocul Immunol Inflamm 2007, 15:99-104. 
